\title{
Free vibration analysis on a simply-supported rhombus plate by using
}

\section{transfer matrix method}

\author{
Liu Can-li* , Yuan Li-yun, Wang Jun-peng , Cao Zhen-qiang, Sun run
}

Department of Automotive and Transportation, Guangxi University of Science and Technology,

Guangxi, Liuzhou , China 545006

aE-mail:1977811882@qq.com, bE-mail: flowlily@126.com

\begin{abstract}
Keywords : rhombus plate; transfer matrix method; perturbation method; a first order ordinary differential matrix equation
\end{abstract}

\begin{abstract}
In this paper, the transfer matrix method is firstly used to analyze the free vibration of a simply-supported rhombus plate. Firstly, using coordinate transformation formula, the solution domain for a rhombus plate is converted into a rectangular one, and the governing equations and the corresponding boundary conditions for the plate in the transformed rectangular domain are obtained. Then, combined with the Fourier series expansion and the perturbation theory, the equation is written in a first order ordinary differential matrix form. Next, the transfer matrix method is used to solve the matrix equations, in order to get the natural frequencies of the free vibration plate. Finally, numerical results are compared with the ones obtained by the finite element method. Example results show that when the difference between the rhombus angle and the corresponding rectangular angle is in a small range, the transfer matrix method proposed in this paper can be applied to analyze the dynamic characteristics of a rhombus plate.
\end{abstract}

\section{Introduction}

Bending vibration analysis of elastic thin plates with regular shape, such as a rectangular plate or a circular one, is easy to carry out analytically. But for the vibration analysis of an irregular plate, such as a rhombus one, numerical methods are generally used instead of analytical one. Because analytical results satisfied boundary condition is difficult to achieve. Among them, finite element method has the wider applicability and higher accuracy. But it is not convenient to carry out parametric analysis due to the need for re-modeling with different parameters. Combined with the approximate function method, variation method is also used to discuss the vibration of thin plates. Ref.[1] carried out the analysis on a rhombus plate by using Kantorovich variation method, but the results was only limited to the uniform loading condition case, and the accuracy was dependent with the amount of higher order approximate functions. Yang and Peng [2] applied the GD method to study the static characteristic for a rhombus plate, which was proposed from the rigorous mathematical principle and with high precision and could be developed to handle the dynamic analysis for the plate. But the truncation error occurred by using the difference of the deflection instead of the differential one. Hence, convenient methods with high accuracy are still pursued for the dynamic problem of an arbitrary shape plate.

In 1920s, transfer matrix method was developed for the dynamic analysis on structures with 
regular shape [3]. Xiang [4] proposed a precise integration method to improve the accuracy of transfer matrix method. However, when applying transfer matrix method, the dynamic governing equation for the structure needed to be written in a form of first order ordinary differential equations. Hence, it was limited to analysis on regular shape structures [5].

In this paper, the free vibration characteristic analysis of a simply-supported rhombus plate is demonstrated by using transfer matrix method. Firstly, the solution domain of a rhombus plate is transformed into a corresponding rectangular one by using the coordinate transformation formula. Then applying the perturbation theory, ignoring the minor items of the equation, and using the Fourier series expansion and dimensionless process, the first order ordinary differential matrix equation for a rhombus plate is achieved, and next is solved by using high precision transfer matrix method. Numerical examples are illustrated to show the accuracy for the method finally.

\section{Governing equations of a rhombus plate}

Considering an isotropic homogeneous thin rhombus plate with thickness $h$, one sets up the coordinate system in its mid-plane. Denotes $u, v, w$ as the displacement components of an arbitrary point at the plate in the $x, y$ and $z$ directions respectively, the corresponding mid-plane displacement components are denoted as $u_{0}, v_{0}, w_{0}$ respectively. According to thin plate theory, the relationship of them can be written as:

$$
u=u_{0}-z \frac{\partial w}{\partial x}, v=v_{0}-z \frac{\partial w}{\partial y} \quad, \quad w=w_{0}
$$

The assumption difference between Ref.[6] and this paper is that the displacements of the middle surface for a plane in the Eq.(1) are not zeros.

Under harmonic excitation condition, the physical quantity in each equation can be expanded into the form of the product of the amplitude and the harmonic excitation factor $e^{j \omega t}$, that is

$$
f(x, y, t)=f(\rho x, y, \omega) e^{j \omega t}
$$

Hence, the internal force- displacement relationship under harmonic excitation condition can be expressed as

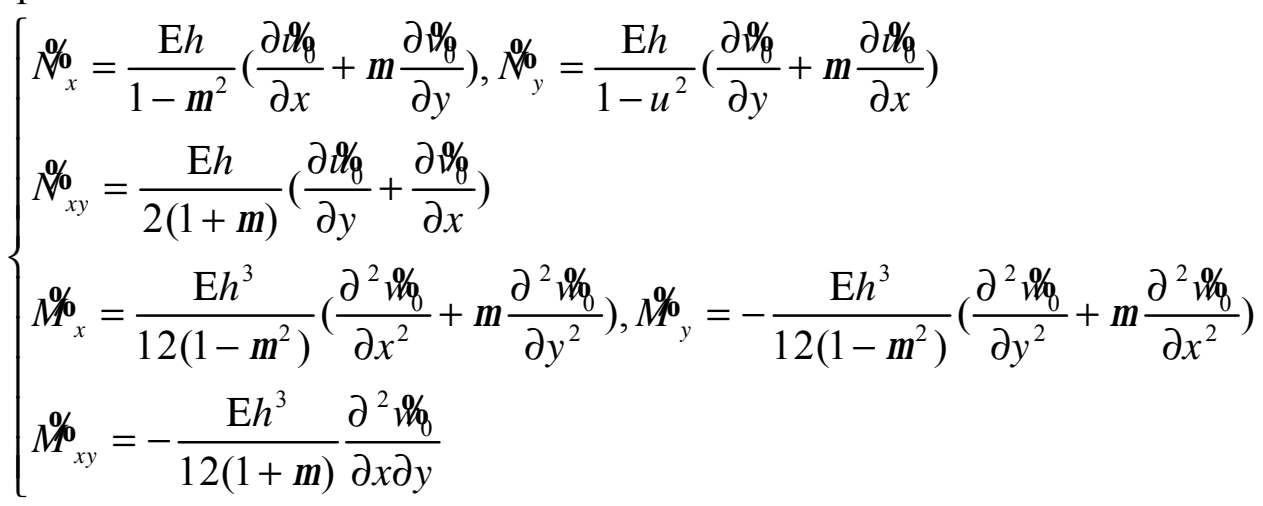

Where $E$ and $\mu$ are the elastic modulus and Poisson's ratio for a plate respectively; $\Re_{x}^{\circ}$ and $\Re_{y}^{\circ}$ are the amplitudes of the mid-plane membrane internal forces for unit length in the $x$ and $y$ direction respectively; $K_{x y}^{\circ}$ is the amplitudes of the mid-plane membrane shear for unit length; $\stackrel{M}{x}_{x}^{\circ}$ and $\stackrel{\%}{y}_{y}$ are the 
mid-plane bending moment amplitudes for unit length in the $x$ and $y$ direction respectively; $\stackrel{\%}{x y}_{x}$ is the mid-plane torque amplitude for unit length .

Meanwhile, the motion equation for the plate under harmonic excitation is written as[7]

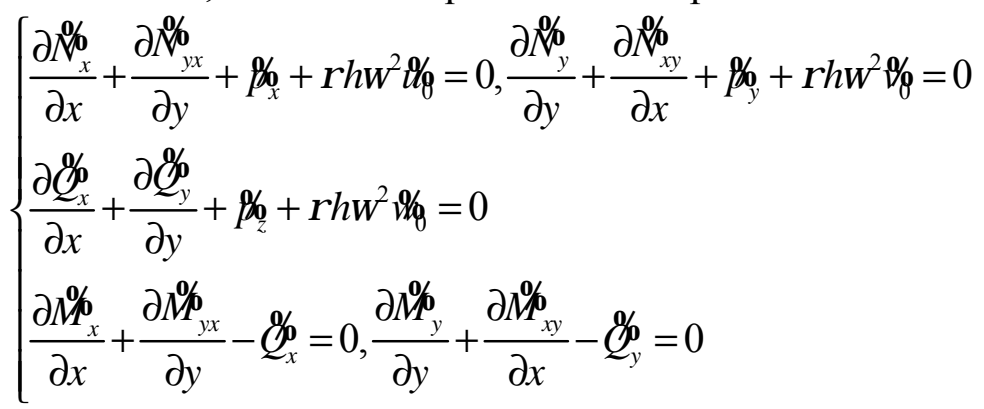

Where, $\mathscr{Q}_{x}^{\circ}$ and $\mathscr{Q}_{y}^{\circ}$ are the mid-plane transverse shear amplitudes for the unit length in the $x$ and $y$ direction respectively; $\wp_{0}(i=x, y, z)$ is the unit area body force amplitude.

In order to utility the boundary conditions conveniently, Kelvin-Kirchhoff equivalent shea-r force amplitudes $S_{x}^{g / c}$ and $V_{x}^{/ /}$are introduced

$$
S_{x}^{\%}=N_{x y}^{\%}, \quad V_{x}^{\%}=Q_{x}^{\circ}+\frac{\partial M \%_{x y}}{\partial y}
$$

Moreover, the solution domain for a rhombus plate is converted into the rectangular one by using coordinate transformation. Denotes $\beta$ as the difference between the rhombus angle and the corresponding transformed rectangular angle, as shown in Fig.1, the relationship between oblique coordinates of $(x, y)$ and Cartesian coordinates of $(\xi, \eta)$ is written as

$$
\xi=x+y \sin \beta, \eta=y \cos \beta
$$

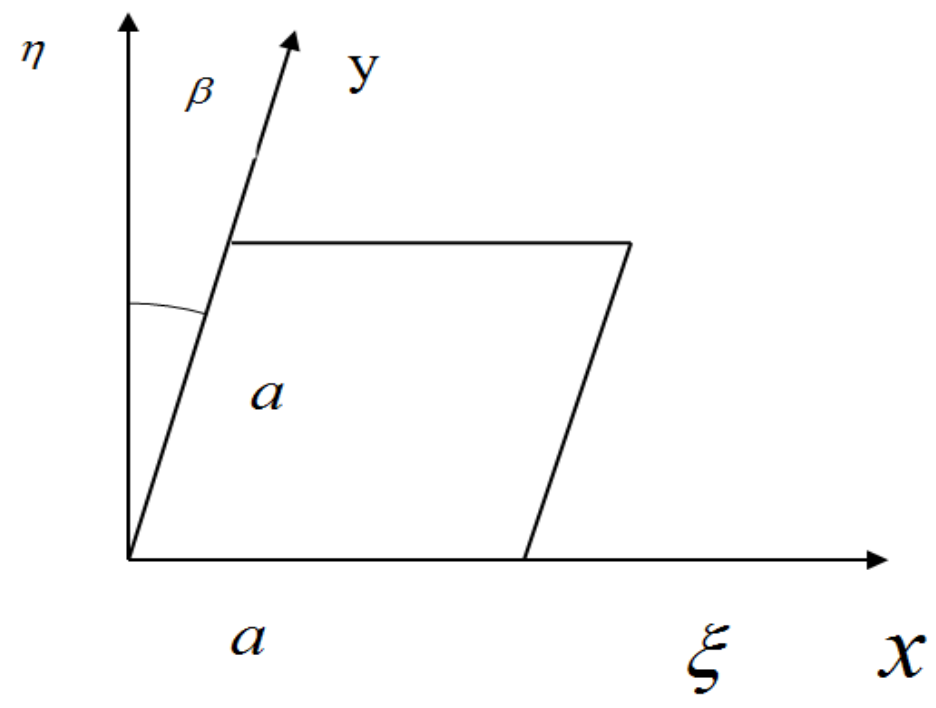

Fig. 1 Transformation between two different Coordinate According to the chain principal of derivation, one achieve 


$$
\frac{\partial}{\partial x}=\frac{\partial}{\partial \xi}, \frac{\partial}{\partial y}=\frac{\partial}{\partial \xi} \sin \beta+\frac{\partial}{\partial \eta} \cos \beta
$$

Combined Eqs.(3-5) and Eq.(7), the internal force-displacement relationship for the plate in the transformed rectangular domain can be written as

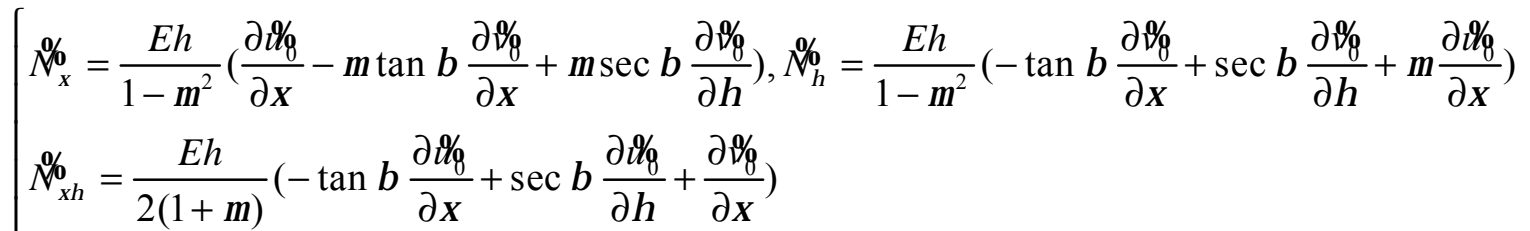

$$
\begin{aligned}
& \left\{M_{\xi}=-\frac{E h^{3}}{12\left(1-\mu^{2}\right)}\left(\left(1+\mu \tan ^{2} \beta\right) \frac{\partial^{2} \mathscr{W} \rho}{\partial \xi^{2}}-2 \mu \tan \beta \sec \beta \frac{\partial^{2} \mathscr{Q} \rho}{\partial \xi \partial \eta}+\mu \sec ^{2} \beta \frac{\partial^{2} \mathscr{Q} \rho}{\partial \eta^{2}}\right)\right. \\
& M_{\eta}^{\%}=-\frac{E h^{3}}{12\left(1-\mu^{2}\right)}\left(\left(\mu+\tan ^{2} \beta\right) \frac{\partial^{2} \mathscr{Q} \rho}{\partial \xi^{2}}-2 \tan \beta \sec \beta \frac{\partial^{2} \mathscr{\wp} \rho}{\partial \xi \partial \eta}+\sec ^{2} \beta \frac{\partial^{2} \mathscr{Q} \rho}{\partial \eta^{2}}\right) \\
& M_{\xi \eta}^{\circ}=-\frac{E h^{3}}{12(1+\mu)}\left(-\tan \beta \frac{\partial^{2} \mathscr{Q} \rho}{\partial \xi^{2}}+\sec \beta \frac{\partial^{2} \mathscr{Q} \%}{\partial \xi \partial \eta}\right)
\end{aligned}
$$

Simultaneously, the motion equations in the rectangular domain can be achieved as

$$
\left\{\begin{array}{l}
\frac{\partial N_{\xi}^{\circ}}{\partial \xi}-\tan \beta \frac{\partial N_{\eta \xi}^{\circ}}{\partial \xi}+\sec \beta \frac{\partial N_{\eta \xi}^{\circ}}{\partial \eta}+P_{\xi}+\rho h \omega^{2} \mathscr{\%} \rho=0 \\
-\tan \beta \frac{\partial N_{\eta}^{\circ}}{\partial \xi}+\sec \beta \frac{\partial N_{\eta}^{\circ}}{\partial \eta}+\frac{\partial N_{\xi \eta}^{\circ}}{\partial \xi}+P_{\eta}+\rho h \omega^{2} \vartheta \%=0 \\
\frac{\partial Q_{\xi}^{\circ}}{\partial \xi}-\tan \beta \frac{\partial Q_{\eta}^{\circ}}{\partial \xi}+\sec \beta \frac{\partial \mathscr{Q}_{\eta}^{\circ}}{\partial \eta}+P_{z}+\rho h \omega^{2} \mathscr{q}_{\rho}^{\circ}=0 \\
\frac{\partial M_{\xi}^{\circ}}{\partial \xi}-\tan \beta \frac{\partial M_{\eta \xi}^{\circ}}{\partial \xi}+\sec \beta \frac{\partial M_{\eta \xi}^{\circ}}{\partial \eta}-Q_{\xi}^{\circ}=0 \\
-\tan \beta \frac{\partial M_{\eta}^{\circ}}{\partial \xi}+\sec \beta \frac{\partial M_{\xi \eta}}{\partial \eta}+\frac{\partial M_{\xi \eta}^{\circ}}{\partial \xi}-Q_{\eta}^{\circ}=0
\end{array}\right.
$$

And equivalent shear force is given as

$$
S_{\xi \eta}^{\%}=N_{\xi \eta}^{o}, \quad V_{\xi}^{\%}=Q_{\xi}^{o}-\tan \beta \frac{\partial M_{\xi \eta}^{\%}}{\partial \xi}+\sec \beta \frac{\partial M_{\xi \eta}^{\%}}{\partial \eta}
$$

Since Eqs.(8-10) are partial differential equations, they cannot be directly solved out analytically. When $\beta$ is small enough, which means that $\tan \beta \rightarrow 0$, the terms involved with $\tan \beta$ can be ignored. Then, by using the perturbation theory and taking $\tan \beta$ as the perturbation term, every physical variable $f$ in Eq.(8-10) is expanded into the form

$$
f=f_{0}+f_{1} \tan \beta+f_{2} \tan ^{2} \beta+\mathrm{L},
$$

And only remain the first term, the others are tiny enough to be ignored. Unless special stated, all the physical variables are the remained term after truncation. And for convenience, the lower subscripts are omitted. 
Next, expanding the remained terms with the Fourier series along the $\eta$ direction, and taking the dimensionless procedure (introducing dimensionless variable $r=\frac{\xi}{L}, L=a$ ) of each remained, that is

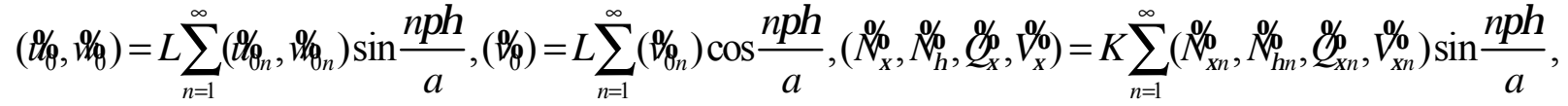

$$
\begin{aligned}
& \left(N_{\xi \eta}^{\circ}, N_{\eta \xi}^{o}, \mathscr{Q}_{\eta}^{\circ}, S_{\xi}^{o}\right)=K \sum_{n=1}^{\infty}\left(N_{\xi \eta n}^{\circ}, N_{\eta \xi n}^{o}, \mathscr{Q}_{\eta n}^{\circ}, S_{\xi n}^{\circ}\right) \cos \frac{n \pi \eta}{a},\left(M_{\xi}^{\circ}, M_{\eta}^{\circ}\right)=(L K) \sum_{n=1}^{\infty}\left(M_{\xi n}^{\circ}, M_{\eta n}^{\circ}\right) \sin \frac{n \pi \eta}{a},
\end{aligned}
$$

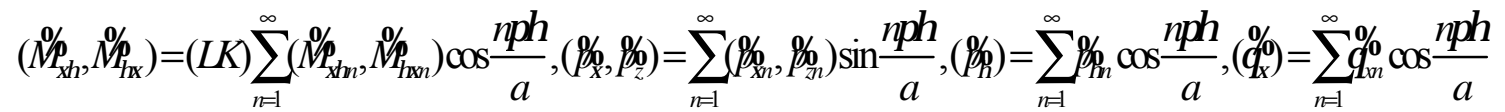

The internal force -displacement relationship equations for the plate are written in an ordinary form,

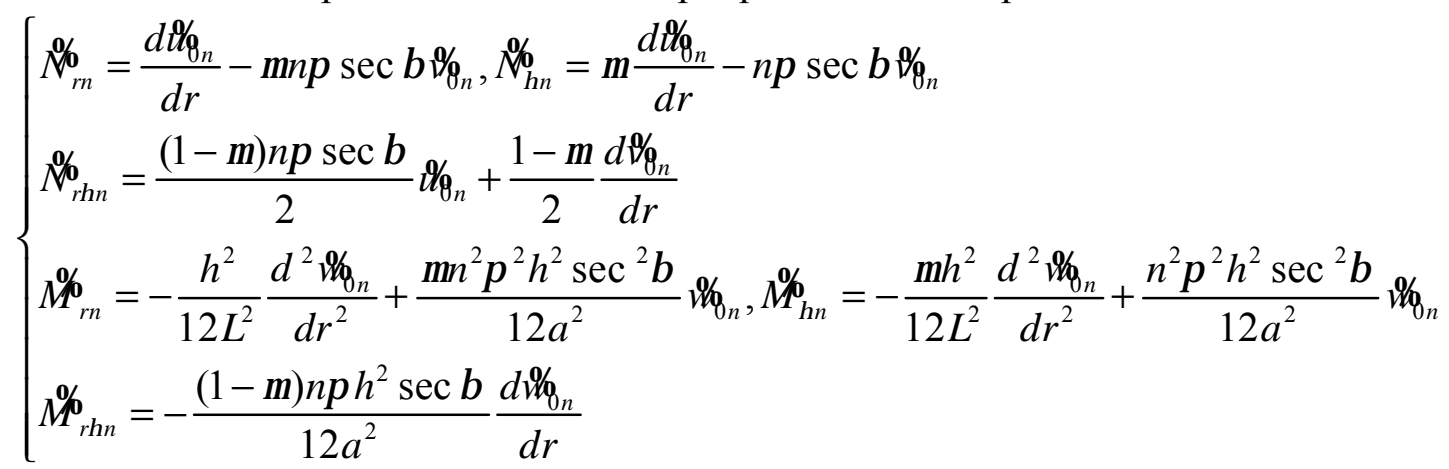

And the motion equations in ordinary form are

$$
\begin{aligned}
& \int \frac{d N_{r n}^{0}}{d r}-n \pi \sec \beta \Re_{\eta r n}^{0}+\frac{L}{K} \beta_{r n}^{0}+\frac{\rho h \omega^{2} L^{2}}{K} \%_{g_{n}}=0 \\
& \left\{n \pi \sec \beta \mathscr{N}_{\eta n}^{0}+\frac{d \mathscr{N}_{m}^{0}}{d r}+\frac{L}{K} \dddot{P}_{\eta n}+\frac{\rho h \omega^{2} L^{2}}{K} \wp_{\phi_{n}}=0\right. \\
& \frac{d \mathscr{Q}_{r n}^{0}}{d r}-n \pi \sec \beta \mathscr{Q}_{\eta n}^{0}+\frac{L}{K} \beta_{z n}^{0}+\frac{\rho h \omega^{2} L^{2}}{K} \mathscr{W}_{g_{n}}=0 \\
& \frac{d M_{r n}^{0}}{d r}-n \pi \sec \beta M_{\eta r n}^{0}-Q_{r n}=0, n \pi \sec \beta M_{\eta n}^{0}+\frac{d M^{0}{ }_{m n}^{0}}{d r}-Q_{\eta n}=0
\end{aligned}
$$

The equivalent shear forces are

$$
S_{r n}^{\circ}=\stackrel{\%}{o}, \quad V_{r n}^{\circ}=\mathscr{Q}_{r n}^{\circ}-n \pi \sec \beta M_{m n}^{\circ}
$$

Similarly, the Fourier series expansion component of the non-dimensional rotation angle is given as

$$
\theta_{r n}^{\%}=-\frac{d \%_{r}^{\circ}}{d r}
$$

Combining Eqs.(12-15), and eliminating other intermediate variables, the integrated first-order ordinary differential matrix governing equation for a thin rhombus plate in corresponding transformed rectangular domain can be derived

$$
\frac{d Z}{d r}=B Z+F
$$

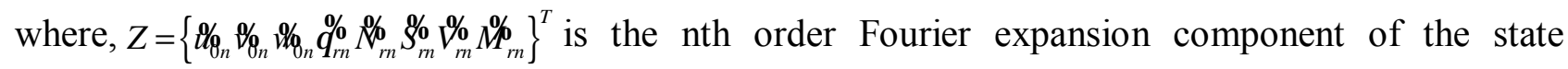
vector; $B$ is the corresponding coefficient matrix; The non-zero components of $B$ are 
$B_{12}=\mu n \pi \sec \beta, B_{15}=1, B_{21}=-n \pi \sec \beta, B_{26}=\frac{2}{(1-\mu)}, B_{34}=-1, B_{43}=-\mu n^{2} \pi^{2} \sec ^{2} \beta, B_{48}=\frac{12 L^{2}}{h^{2}}$

$B_{51}=-\frac{\rho h \omega^{2} L^{2}}{K}, B_{56}=n \pi \sec \beta, B_{62}=-\frac{\rho h \omega^{2} L^{2}}{K}+\left(1-\mu^{2}\right) n^{2} \pi^{2} \sec ^{2} \beta, B_{65}=-\mu n \pi \sec \beta$,

$B_{73}=-\frac{\rho h \omega^{2} L^{2}}{K}+\frac{\left(1-\mu^{2}\right) n^{4} \pi^{4} h^{2} \sec ^{4} \beta}{12 a^{2}}, B_{78}=\mu n^{2} \pi^{2} \sec ^{2} \beta, B_{84}=\frac{(1-\mu) n^{2} \pi^{2} h^{2} \sec ^{2} \beta}{6 a^{2}}, B_{87}=1$,

and $F=\frac{L}{K}\left\{0,0,0,0, \not \%_{r m}^{\circ}, \not \%_{\eta n}, \not p_{z n}, 0\right\}^{T}$ is the integrated load vector

\section{Solution method of the governing equation}

In this paper, the free vibration characteristics for a rhombus plate are studied, i.e. $F$ is a zero vector in Eq.(17). Then, by adopting the transfer matrix method, one gets the transfer relationship between the vectors at the two boundary ends

$$
Z_{1}=C Z_{0}, C=e^{B\left(r_{1}-r_{0}\right)}
$$

where $Z_{0}$ and $Z_{1}$ are the state vectors at the initial boundary $\left(r_{0}=0\right)$ and ending boundary $\left(r_{1}=1+\sin \beta\right)$ respectively, $C$ is the transfer matrix.

By using coordinate transformation formula, the solution domain with a simply-supported rhombus plate is converted into the rectangular one, and the boundary condition in the rectangular domain is rewritten as

$$
r=0,1+\sin \beta: \quad u_{0 n}=0, S_{r n}=0, w_{0 n}=0, M_{r n}=0,
$$

Substituting the boundary condition into Eq.(19), after manipulation, on gets

$$
D\left[\begin{array}{c}
v_{s n 0} \\
\theta_{c n 0} \\
N_{c n 0} \\
V_{c n 0}
\end{array}\right]=\left[\begin{array}{l}
0 \\
0 \\
0 \\
0
\end{array}\right], D=\left[\begin{array}{llll}
C_{12} & C_{14} & C_{15} & C_{17} \\
C_{32} & C_{34} & C_{35} & C_{37} \\
C_{62} & C_{64} & C_{65} & C_{67} \\
C_{82} & C_{84} & C_{85} & C_{87}
\end{array}\right]
$$

Herein, $D$ is the characteristic matrix, in which the subscript $i, j$ of $C_{i j}$ represent the $i$ th row

and the $j$ th column element in matrix $C$.

When all the physical parameters for the plate are known, the determinant of matrix $D$ will be dependent on excitation frequency. For the free vibration at the natural frequencies, the determinant of matrix $\mathrm{D}$ will be equal to zero, that is

$$
|D|=0
$$

Eq.(22) is the characteristic equation for the free vibration of the rhombus plate. By solving Eq.(22), the circular natural frequencies $\omega$ for the plate can be obtained, the program is designed by MATALAB. Then, the vibration natural frequencies for a rhombus plate are obtained

$$
f=\frac{\omega}{2 \pi}
$$




\section{Numerical simulations}

In order to verify the validity of the method in this paper, we will take a thin plate with a small angle as an example.

Considering the simply-supported rhombus plate with geometric parameters $2.00 m \times 2.00 m \times 0.02 m, \beta=\pi / 36$, its physical parameters are listed in Table 1 .

Table.1 Physical parameters of the rhombus Plate

\begin{tabular}{ccc}
\hline \hline Density $\rho\left[\mathrm{kg} / \mathrm{m}^{3}\right]$ & $\begin{array}{c}\text { Elasticity } \\
\text { modulus } E[\mathrm{~Pa}]\end{array}$ & $\begin{array}{c}\text { Poisson's } \\
\text { ratio } \mu\end{array}$ \\
\hline 7850 & $1.9 \times 10^{11}$ & 0.305 \\
\hline
\end{tabular}

By using the presented method and the finite element method respectively, the natural frequency with order $(1,1)$ is obtained as shown in Figure 2 and figure 3.

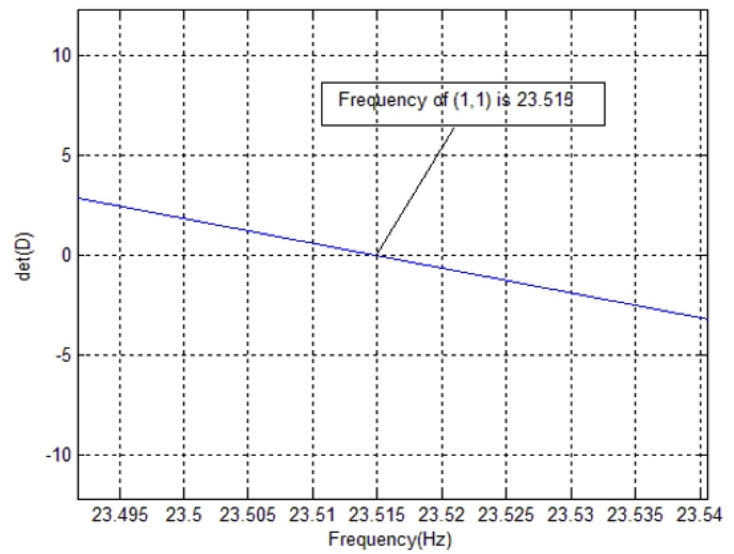

Fig.2 Frequency with order $(1,1)$

by the presented method

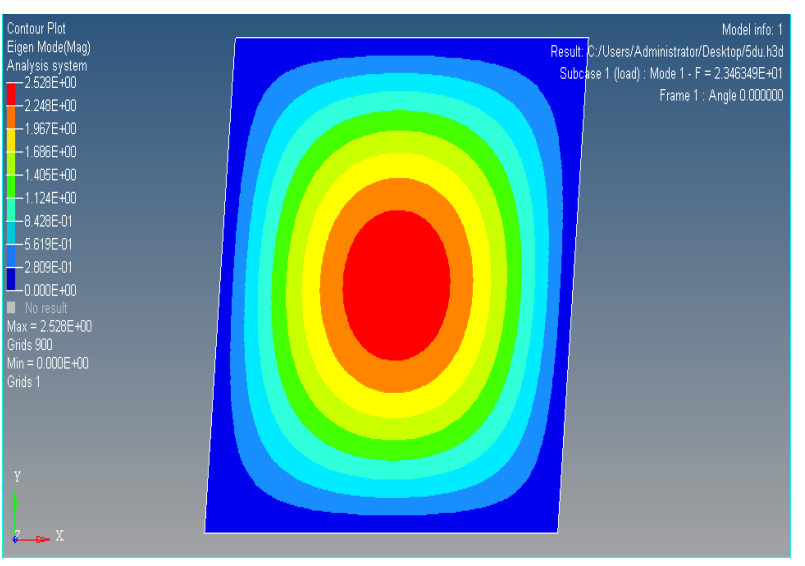

Fig. 3 Vibration modal with order $(1,1)$

by the finite element method

The other natural frequencies achieved by the presented method and the finite element method are given in Table 2. It shows that the relative difference of the natural frequencies obtained between the two methods is limited to $3 \%$. So it proved that the transfer matrix method proposed in this paper could gain enough precision.

Tab.2 The comparison of the solution between the article and the finite element

\begin{tabular}{cccc}
\hline \hline$[\mathrm{m}, \mathrm{n}]$ & $\begin{array}{c}\text { The presented method } \\
f[\mathrm{~Hz}]\end{array}$ & $\begin{array}{c}\text { Finite element } \\
\text { solution } \\
f_{s}[\mathrm{~Hz}]\end{array}$ & $\begin{array}{c}\text { Relative } \\
\text { difference } \\
{[\%]}\end{array}$ \\
\hline$(1,1)$ & 23.515 & 23.463 & 0.22 \\
$(2,1)$ & 58.644 & 57.252 & 2.4 \\
$(1,2)$ & 58.922 & 60.186 & 2.1 \\
$(2,2)$ & 94.085 & 93.129 & 1.0 \\
\hline \hline
\end{tabular}

As mentioned in section 2, the governing equation are truncated by omitting the higher order perturbation terms involved with $\tan \beta$. Hence, the precision of the presented method will dependent on angle $\beta$ obviously. When $\beta$ is big enough, the presented method would turn to be incorrect. So it is necessary to consider the influence of angle $\beta$ on the accuracy of the presented 
method. Numerical example 2 is demonstrated in Table. 3 when $\beta$ is changed while other parameters are remaining unchanged, the relative difference between the presented method and the finite element method. Due to the limited space, only frequencies obtained with some angles (that is $\beta=\pi / 36, \beta=\pi / 18, \beta=\pi / 9, \beta=\pi / 6$ respectively) are not listed.

It is shown that as the angle $\beta$ increases, the relative difference of the frequencies between the presented method and the finite element method is gradually increasing on the whole. Moreover, the higher the order is, the greater the difference is. When the angle is less than $\pi / 9$, the relative difference could be limited to $5 \%$, which would be accurate enough.

Tab.3 Comparison of the natural frequencies between the presented method and finite element method with different angles $\beta$

\begin{tabular}{ccccc}
\hline \hline Angle & $\begin{array}{c}\text { Modal } \\
\text { order } \\
{[\mathrm{m}, \mathrm{n}]}\end{array}$ & $\begin{array}{c}\text { The presented } \\
\text { method } \\
f[\mathrm{~Hz}]\end{array}$ & $\begin{array}{c}\text { Finite element } \\
\text { method } \\
f_{s}[\mathrm{~Hz}]\end{array}$ & $\begin{array}{c}\text { Relative } \\
\text { difference } \\
{[\%]}\end{array}$ \\
\hline \multirow{2}{*}{$\pi / 36$} & $(1,1)$ & 23.511 & 23.463 & 0.23 \\
& $(2,2)$ & 94.058 & 93.129 & 1.0 \\
$\pi / 18$ & $(1,1)$ & 23.789 & 23.895 & 0.44 \\
& $(2,2)$ & 95.153 & 93.066 & 2.2 \\
$\pi / 9$ & $(1,1)$ & 24.975 & 25.742 & 3.0 \\
& $(2,2)$ & 99.902 & 94.256 & 6.0 \\
$\pi / 6$ & $(1,1)$ & 27.33 & 29.329 & 6.8 \\
& $(2,2)$ & 109.312 & 98.998 & 10 \\
\hline \hline
\end{tabular}

\section{Conclusions}

Based on using the transfer matrix method and perturbation theory, the paper proposes a new method to analyze the dynamic characteristics for a rhombus plate. Example results show that the natural frequency obtained by the transfer matrix method and the finite element method is accurate enough in a small range ( $\beta$ is less than $\pi / 9$ ), which could be a convenient means for the parametric study on rhombus plates.

\section{Acknowledgement}

This work is supported by the National Natural Science Foundation of China through Grant No. 11502056 and No.51665006, the National Natural Science Foundation of Guangxi Province through Grant No. 2015GXNSFBA139007, the Key Laboratory of Open Foundation of Guangxi Province through Grant No. 1404544, and the Doctoral program of Guangxi science and Technology University through Grant No. $12 \mathrm{Z} 09$.

\section{Reference}

[1] Wang,L., Kantorovich method of the bending parallelogram plate[J].Acta Mechanica Solida Sinica, 1983,4(3):420-426.

[2] Yang,L. and Peng,J., GD method for solving the bending problem of the bending parallelogram 
plate[J]. Journal of Chengdu University, 2014, 9(3):230-233.

[3] Zhu,J.Q., The application of transfer matrix method in the calculation of structural vibration[J]. Earthquake engineering and Engineering Vibration, 1984, 4(1):36-44.

[4] Xiang,Y., The exact form of transfer matrix of analyzing the free vibration of structures[J]. Journal of Vibration and shock, 1999, 18(2):69-74.

[5] Wu,H.J.,Xiang,Y. and Yuan,L.Y., Analysis of vibration and damping characteristics of PCLD beam[J]. Journal of Guangxi University of science and technology, 2014, 12(4):40-45.

[6] Xu,Zh.L., mechanics of elasticity[M].Beijing: Higher Education Press, 2006(in Chinese).

[7] CAO Z.Y. Vibration Theory of Plate and Shell [M].Beijing: China Railway Publishing House, 1989 (in Chinese). 